\title{
Parent-Child Autonomic Synchrony During Vicarious Extinction Learning in Pediatric PTSD
}

\author{
Running Title: Vicarious Parent-Child Synchrony in Pediatric PTSD \\ Grace C. George MPA a,b,c, Sara A. Heyn JD PhDc, Shuka Konishic, Marie-France Marin PhDd, \\ Mohammed R. Milad PhD ${ }^{\mathrm{e}}$, Ryan J. Herringa MD PhD ${ }^{\mathrm{b}, \mathrm{c}}$ \\ a Neuroscience \& Public Policy Program, University of Wisconsin-Madison, Madison, WI, USA \\ b Neuroscience Training Program, University of Wisconsin-Madison, Madison, WI, USA \\ c Department of Psychiatry, BRAVE Youth Lab, 6001 Research Park Blvd., Madison, WI \\ 53719, USA \\ d Department of Psychology, Université du Québec à Montréal, Montreal, Quebec, Canada \\ e Department of Psychiatry, New York University, New York \\ ACKNOWLEDGEMENTS
}

\begin{abstract}
We would like to thank the Brave Research Lab with their help in recruitment and data collection for this study and their feedback on the results. We also want to extend our genuine gratitude to the families and youth who have participated in our study.

Corresponding author at: Department of Psychiatry, BRAVE Youth Lab, 6001 Research Park Blvd., Madison, WI 53719, USA. E-mail address: gcgeorge@wisc.edu (Grace C. George)
\end{abstract}

Abstract: 225

Main Text: 3245

Figures: 3

Tables: 1 
Abstract

31 Children must learn basic functional processes directly from their caregivers and child psychopathology may disrupt this transmission. This transmission may be seen through biological measures like peripheral nervous system outputs like skin conductance (SCR). Fear learning deficits have been seen in affective disorders like PTSD and are useful for studying parent-child learning transmission. Our study uses a vicarious fear extinction paradigm to study if biological synchrony (SCR and heart rate variability $(\mathrm{HRV}))$ are potential mechanisms in which children learn safety cues from their parents. There were 16 dyads (PTSD $n=11, T D n=5)$ undergoing a vicarious fear extinction paradigm. We used cross-recurrence quantification analysis (CRQA) to assess SCR and HRV synchrony between parent-child dyads. We then used a linear model looking at group differences between PTSD dyads and typically developing (TD) dyads. For SCR, we saw a significant group difference $(p=.037)$ indicating that TD dyads had higher SCR synchrony compared to PTSD dyads. For HRV, there were no group differences between PTSD and TD dyads $(p=.325)$. These results suggest that SCR synchrony, but not HRV, may be a potential mechanism that allows for fear and safety learning in youth. While this is preliminary, it may give the first insights on how therapies such as Trauma-Focused Cognitive Behavioral Therapy critically rely on parental coaching to model appropriate fear responses to help their child to recover from trauma. 
$\underline{\text { Introduction }}$

Fear extinction learning has been widely used to understand affective disorders like anxiety, depression, and post-traumatic stress disorder (PTSD; Herringa et al., 2013; Milad et al., 2014). In PTSD, previous studies have indicated disruptions in proper fear extinction learning like enhanced acquisition, stimulus generalization, and impaired extinction learning (for a review see Milad et al., 2014). While this has been studied more frequently in adults (Blechert et al., 2007; Helpman et al., 2016; Milad et al., 2008), few have looked at how extinction learning may be altered in youth with PTSD (Heyn et al., 2022).

Children's ability to learn emotional content from their parents and caregivers is one of the most important pieces of child development (Debiec \& Olsson, 2017). However, when parents or children suffer from internalizing disorders, like PTSD, these signals can be disrupted or difficult to interpret. Having reliable transmission of emotional content is imperative, especially for therapy including trauma focused cognitive behavioral therapy (TF-CBT) which utilizes dyadic treatment to help youth with trauma disorders (Golkar et al., 2016). Therefore, it is important to study how children vicariously learn fear and safety from their caregivers to better understand how to effectively use this transmission. Youth's use of vicarious, or observational, fear learning from their caregivers has been shown to influence their own fear development indicating that this is an important learning mechanism (Rachman, 1977). Previous studies have further shown that parents expressions of fears and anxieties can lead to the development of specific fears in the child (Dunne \& Askew, 2018; Marin et al., 2020). During acquisition of fear, one study found that children that were more sensitive to anxiety and had lower father-child relationship security had increased reactivity to fear conditioning compared to children that did not, demonstrating that psychopathology and relationship quality may both influence vicarious fear learning (Bilodeau-Houle et al., 2020). While fear learning may be enhanced in youth that have anxiety or trauma related disorders, healthy fear extinction or extinction recall may be disrupted, which has also been seen in adults (Marusak et al., 2020; Pitman et al., 2012). Therefore, it is important to study extinction and recall of fear, in addition to acquisition, to fully understand the impact of psychopathology of vicarious fear learning in youth.

One possible mechanism through which vicarious extinction can occur is through parent-child biological synchrony. Synchrony is the temporally-matched coordination of behavior, feelings, or biological responses between two people (Feldman, 2012). For parent-child dyads, synchrony is a critical method of learnt emotion regulation in children and a way to foster healthy attachments (Davis et al., 2017). Physiological synchrony, a subset of biological synchrony, uses peripheral nervous system methods like skin conductance response (SCR), heart rate variability (HRV) or cortisol activity to evaluate the degree to which caregivers and their children are coupled (Feldman, 2012). It has been documented that youth with PTSD tend to have differences in both autonomic and behavioral synchrony with their parents indicating that this may be a potential 
mechanism that may lead to the inability to learn cues from their parent (Feldman, 2007; Motsan et al., 2020). Understanding the biological mechanism behind vicarious learning is crucial for understanding transmission of fear and safety cues between caregivers and children, especially in those with fear related disorders like PTSD.

For this study we will be using a three-day vicarious fear extinction paradigm. During this paradigm, children go through both direct extinction and a vicarious extinction which includes watching their parent undergo direct extinction. While this is a pilot study, preliminary evidence from our main analyses suggest that youth with PTSD have increased arousal (SCR) during vicarious fear learning compared to typically developing youth (Heyn et al., 2022). This demonstrates that there maybe be a biological mechanism at play when youth are learning fear and safety cues from their caregivers, and potentially this is disrupted in youth with PTSD. We want to expand on this finding and understand if biological synchrony during vicarious fear extinction is different in youth with PTSD and if this is related to recall of the fear.

We will be using two biological metrics to assess fear learning: SCR and HRV. SCR is a widely used measure of physiological arousal and is one of the most common biological metrics of fear condition and extinction (Faghih et al., 2015). Another common metric is HRV and has been found to be linked to VmPFC and amygdala modulation which are important in the regulation of fear responses (Milad et al., 2007; Schiller et al., 2008). To measure how well the child is learning from the parent, we will use these two measures to assess biological synchrony between the dyads.

In this study, we will be assessing if there are group differences in biological synchrony during vicarious learning and if this is related to fear extinction recall and dyadic psychopathology symptoms. We predict that PTSD dyads will have lower biological synchrony compared to their TD counterparts. Further, we predict that PTSD related symptoms will be related to synchrony. Lastly, in exploratory analyses, we expect that synchrony will predict biological responses during recall, but only for the vicarious conditioned stimulus and not for either the direct or non-conditioned stimuli.

\section{Methods}

In this pilot study, we had 16 parent-child dyads with youth ranging from ages 717. 11 of those dyads included a child with PTSD and five that were typically developing (TD). Exclusion criteria for our youth participants included past or present brain injury, unstable or sever medical conditions, substance abuse, acute suicidality, or ongoing abuse. Each parent-child dyad was assessed for past and current psychopathology diagnosis, including PTSD status, using the Mini-International Neuropsychiatric Interview Screen (MINI; Sheehan et al., 1998). Further psychopathology questionnaires for the child included the Mood and Feelings Questionnaire (MFQ) for depression, the Screen for Child Anxiety-Related Emotional Disorder (SCARED) for anxiety, and the UCLA PTSD Reaction Index (PTSD-RI) for PTSD symptoms. The PTSD-RI for the 
DSM-V and DSM-IV were given to different participants, and was therefore converted using a validated conversion tool (Cheng et al., 2021).

In the current study, parent-child dyads underwent a three-day vicarious and direct fear learning paradigm. We used an adaptation from Milad and colleagues' (2007) and is described in detail in Heyn et al., 2022 (Milad et al., 2007). Briefly, each dyad completed a fear learning paradigm separately. On day one, both parent and child were conditioned to colored stimuli, for the parent, they had one conditioned stimuli CS+D while the child was conditioned to two different stimuli (CS+D and CS+V). Then on the second day, the parent went through direct extinction while the child went through both direct extinction and vicarious extinction which consisted of watching their parent going through direct extinction. Then the dyad each went through recall on the third day. All three task days were approximately 24 hours apart. For conditioning, we used tactile electrodermal stimulation. Each participant was allowed to manually select their level of stimulation. No participants dropped out of the pilot study due to problems of the stimulation. Further discussion of experimental design can be found in Heyn et al. 2022.

During vicarious learning, we measured SCR, heart rate (HR), and respiration of each dyad. For the synchrony analyses, we used SCR and HRV. For both SCR and $\mathrm{HRV}$, we cut each timeseries at the beginning of the first fixation to the beginning of the last fixation. SCR analyses including a low-pass filter of $1 \mathrm{~Hz}$ and down sampling to 8 $\mathrm{Hz}$ using Ledalab (Benedek \& Kaernbach, 2010). HRV analyses used HR and respiration to create time and frequency domains using MindWare software (MindWare Technologies Inc., Westerville, $\mathrm{OH}$ ).

Statistical analyses were performed in RStudio (RSTudio Team, 2012). For each synchrony analysis, we used cross-recurrence quantification analysis (CRQA) using the R package crqa (Coco \& Dale, 2014). In brief, CRQA captures recurring properties and patterns of two distinct time series. Increased CRQA measures, or synchrony, indicates that the two time-series, for example parent-child SCR, resemble each other or mimic each other over time. We followed Pärnamets et al. (2019) parameters for the CRQA analysis. We then picked three metrics (Determinism, Entropy, and Laminarity) that were highly correlated $r>.90$ and conducted a Principal Component Analysis with varimax rotation to find a single composite score of synchrony using the psych package in R. For our main analyses, we ran a linear regression for group (TD vs PTSD) while covarying for child age and sex. We then ran post-hoc analyses on significant group differences. To ensure that this was due to vicarious learning, we also ran group differences on synchrony when both the child and parent were undergoing direct extinction. We then conducted Pearson correlations between child symptoms from the PTSD-RI, MFQ, SCARED, and synchrony with FDR correction. We further covaried for parent age and lifetime or current psychopathology diagnosis of the parent on the group differences. Lastly, we conducted three exploratory repeated measures analyses to understand if synchrony was related to fear extinction outcomes measures. First, to assess if synchrony was related to fear learning responses, we did a repeated 
measures regression to see if synchrony and CS type predicted extinction retention index (ERI). ERI was calculated by taking the average SCR of the first 2 recall trials and dividing it by the highest SCR during the conditioning trials. Second, we wanted to know if synchrony was related to SCR responses during the first four trials recall and if these responses were moderated by CS type. Lastly, we wanted to understand if synchrony was related to expectancy of the shocks during the first trial of recall and if this was moderated by CS type. All models, besides the correlations, were covaried with child age and sex and Z-scored. Due to the skew of the recall data, all recall SCR data was log transformed and then Z-scored.

\section{$\underline{\text { Results }}$}

There was a significant SCR group synchrony difference between the PTSD and TD groups during vicarious extinction learning $b=1.25, t(13)=2.34, p=.037$ and an effect size of $\eta 2=.31$ (Figure 1). There was no significant difference between groups during direct extinction for SCR synchrony $b=-0.18, t(13)=.35, p=.62$. For HRV, there was no group synchrony differences during vicarious extinction $b=.40, t(13)=1.03$, $\mathrm{p}=.325, \quad \eta 2=.08$.

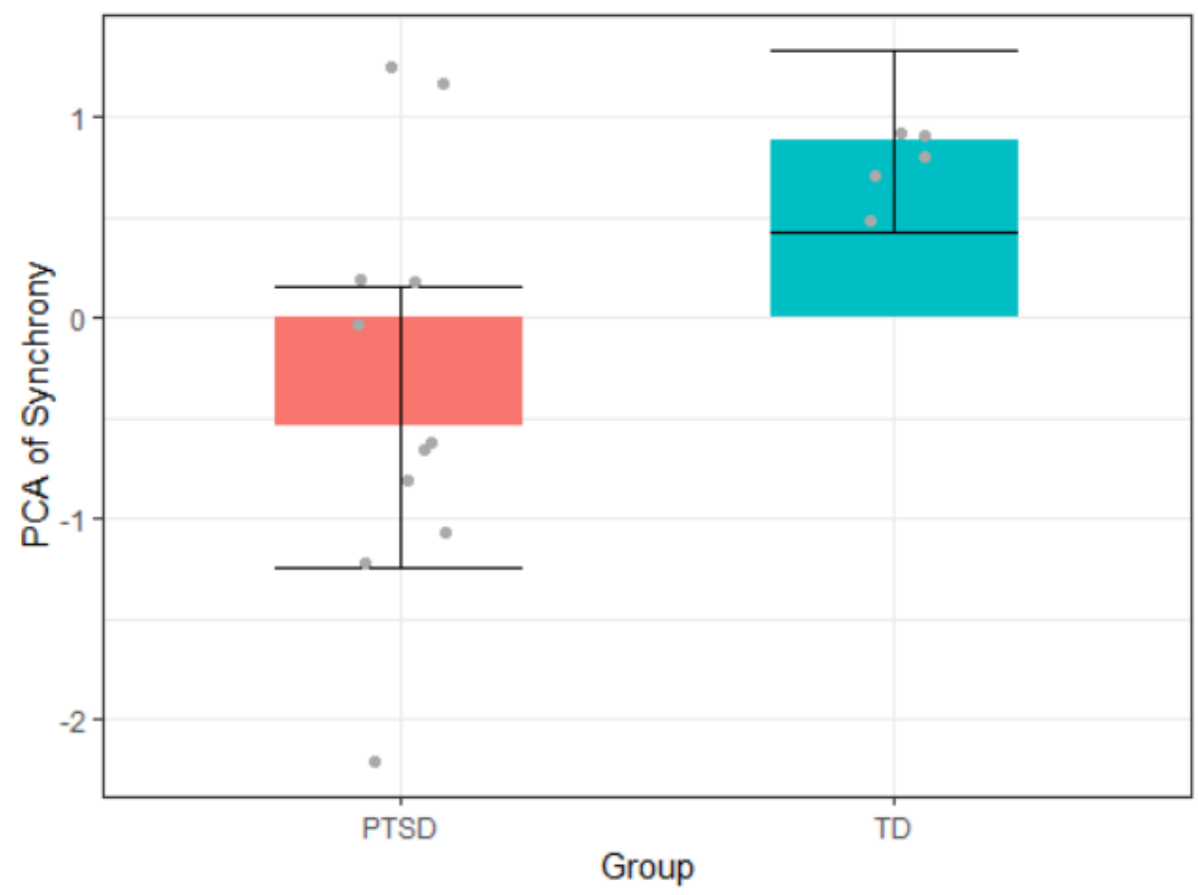

Fig 1. Group synchrony differences between PTSD and TD dyads. Using a linear model, we found a significant group differences between PTSD and TD dyads. Overall, we see that TD dyads showed higher synchrony compared to PTSD dyads.

Due to the group differences, we wanted to test if synchrony was also related to any child symptoms. There were no correlations between synchrony and any of the child symptom measures $p>.27$ (Table 1$)$. When covarying for parent's age $(b=0.72$, 
$t(12)=1.27, p=.23)$, lifetime diagnosis $(b=1.06, t(12)=1.01, p=.08)$, and current diagnosis $(b=1.04, t(12)=1.9, p=.09)$, while group differences went above .05 with parent covariates, the sample size would indicate that likely there group differences would stay with a larger sample size. These results should be taken with caution due to 198 the marginal significance.

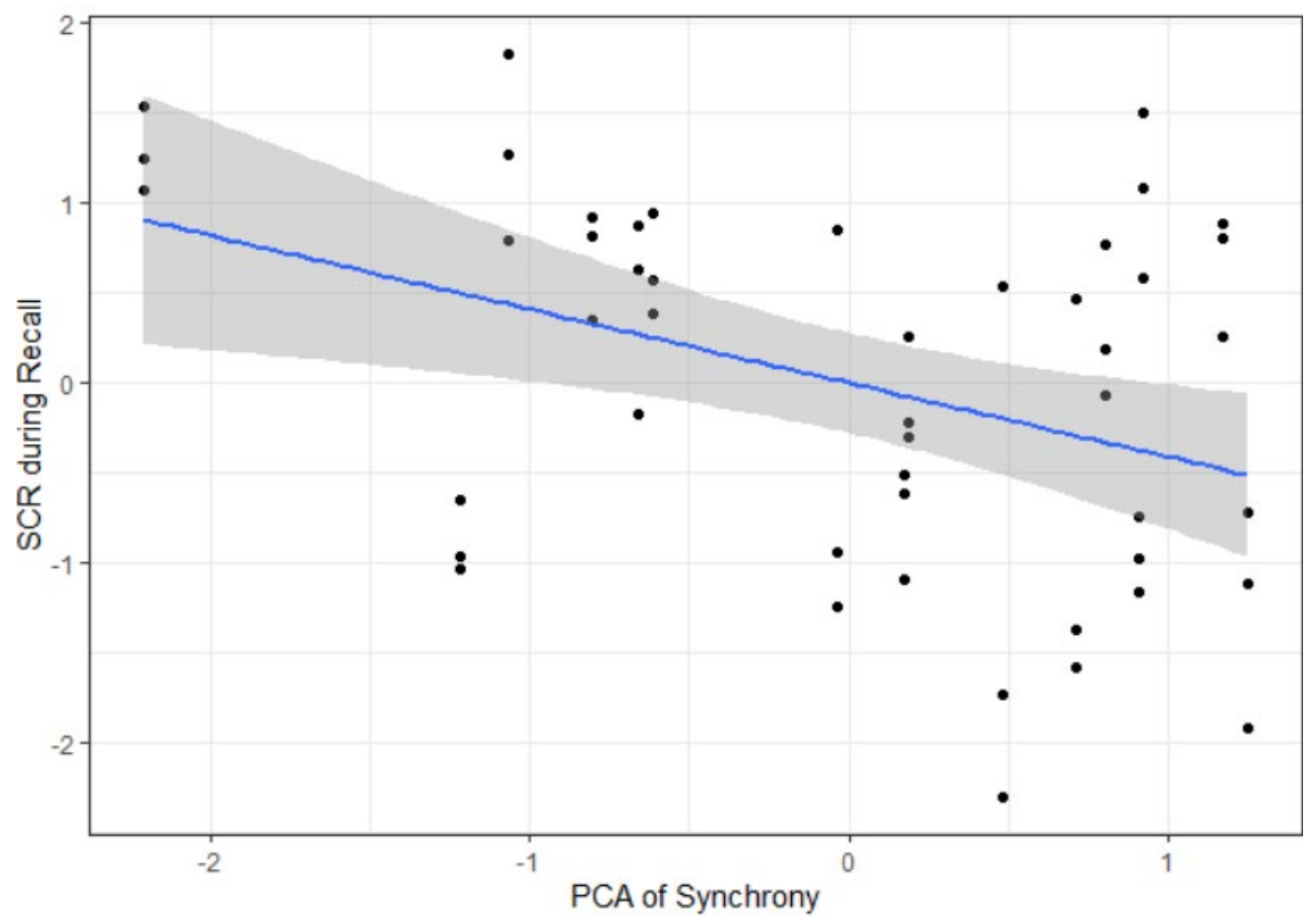

Fig 2. Increased synchrony relationship to decreased SCR during recall. We found a significant main effect of synchrony on SCR during recall indicating that synchrony may be related to overall decreased in arousal, but not CS specific decreases.

For the exploratory analyses, synchrony by CS type and the main effects did not significantly predict ERI, $p>19$. For the second analysis, there was a significant main effect of vicarious SCR synchrony on recall SCR $F(11,12)=4.62, p=0.032$ indicating decreased synchrony was related to increased SCR during recall (Figure 2), but there was no significant interaction ( $p>.9$ ) between synchrony and CS type on SCR during recall. For the third analysis, there was a significant CS type by synchrony interaction $F(11,12)=3.62, p=.02$ for expectancy (Figure 3). We also assessed if any of the CS types were significant from zero. The $C S+D(t(11)=-.77, p=.46)$ and $C S+V(t(11)=1.28$, $\mathrm{p}=.23$ ) were not significantly different from zero, but CS- was marginally significant from zero $(t(11)=-2.02, p=.068)$. 


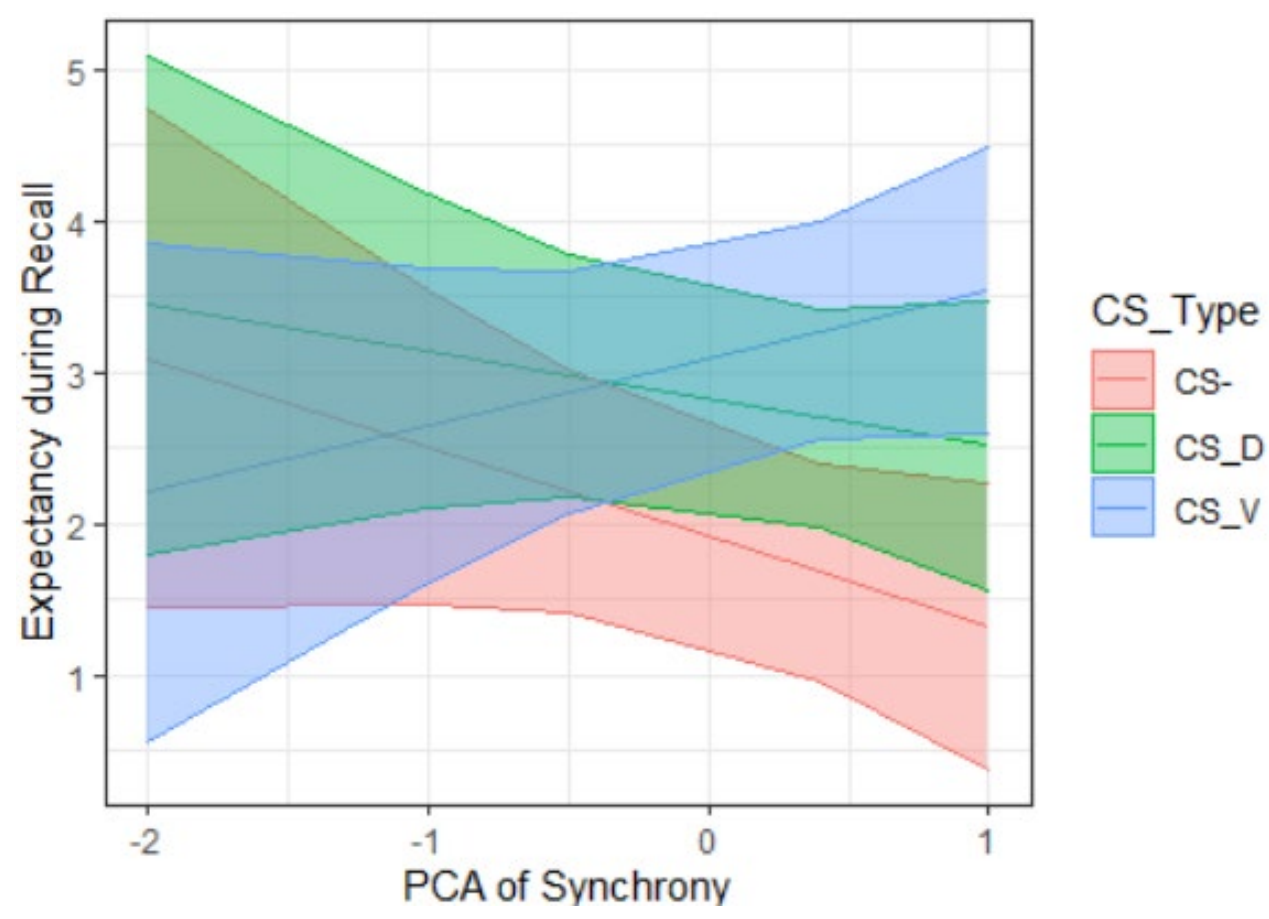

Fig 3. Significant CS type and synchrony interaction predicting expectancy. We found a significant interaction of CS type and synchrony on expectancy during recall. Only the CS- is marginally significant from zero suggesting that synchrony may be a predictor of safety instead of fear learning.

\section{Discussion}

In this pilot study, we explored potential mechanisms that are related to vicarious fear and safety learning, specifically in a difficult group to recruit, youth with PTSD. We hypothesized that synchrony, or the coupling of two biological systems, in this case parent and youth psychophysiological outputs, may be an important mechanism of transmission of cues between each dyad. Overall, we found group differences in SCR synchrony between dyads that have youth with PTSD compared to typically developing dyads. In exploratory analyses, there was preliminary evidence that synchrony may lead, or be related to SCR reactivity during recall indicating the possibility that dyads with higher synchrony during extinction learning have lower overall arousal. Further, synchrony was moderated by CS type to predict expectancy. While both the CS+V and $C S+D$ slopes were not different from zero, for the CS- ( $p=.068)$, increased synchrony was marginally related to less expectancy of a stimulation. This possible indicates that increased synchrony is related to better learning of safety cues.

Overall, we found that youth with PTSD had lower SCR synchrony compared to their TD counterparts (Figure 1). However, we needed to account for the possibility that synchrony was not due to only similar familial reactions to extinction learning, but that it was accurately attributed to physically watching the parent. To account for this, we did the same analysis with the child's direct extinction SCRs and did not find this group 
difference suggesting that it was not general reactions to extinction learning and that vicarious learning specifically was related to changes in autonomic processing.

While we predicted SCR and HRV to be significant in our main analyses, only SCR came back significantly different between groups. Previous studies have shown that SCR synchrony was associated with greater threat learning and that increased SCR synchrony during fear acquisition was related to fear conditioned responses in parent-child dyads (Marin et al., 2020; Pärnamets et al., 2020). Adult PTSD studies have found increased SCRs during recall suggesting a lack of fear extinction, however youth with PTSD has found mixed results indicating that much is still unknown about how youth with psychopathology learn and extinguish fear (Garfinkel et al., 2014; Marusak et al., 2020; McLaughlin et al., 2016; Milad et al., 2009). For example, McLaughlin and colleagues found blunted SCRs to the CS+ and poor differentiation of CS types during the conditioning and extinction in maltreated youth, while Marusak and colleagues found no differences in SCRs between TD and maltreated youth but did see behavioral differences of fear learning. Our pilot study specifically found group differences in SCR during vicarious but not direct extinction learning (Heyn et al. 2022). Social fear learning may be what is truly affected in trauma-related disorders. Our synchrony analyses strengthen that argument, as synchrony, which a known mechanism of learning, was blunted in dyads that had a child with PTSD (Davis et al., 2017).

Currently, there is less evidence to implicate HRV with synchrony and extinction learning. Previous studies looking at HRV synchrony found that it is often found within relationships with positive attributes such as in higher levels of closeness, trust, and prosocial behaviors (Danyluck \& Page-Gould, 2019; Goldstein et al., 1989). Individuals with social anxiety disorder (SAD) were found to have difficulty in creating HR synchrony in more intimate social contexts compared to individuals without SAD, thus leading to the decreased ability in developing relationships (Asher et al., 2021). However, these studies used general play or free-roam behavioral tasks instead of structured fear extinction task like we used. This may lead to differences in our results. Further exploration on youth with PTSD and HRV is needed to understand how or if $\mathrm{HRV}$ is related to vicarious fear extinction.

For our exploratory analyses, we wanted to understand if synchrony could be used to predict specific outcomes of fear extinction learning, ERI, SCR during recall, and expectancy of stimulation, and if this was moderated by CS type (CS+D, CS+V, and CS-). ERI is used as a measure of extinction learning, as it takes into account baseline levels of autonomic reactivity during fear acquisition and compares it to outputs during recall (Milad et al., 2008). We did not see any significant main effects or interactions in this model. While others have shown that there are differences in ERI between combat veterans with PTSD and those without, our results did not support this. However, our results may differ due to our low sample size and our youth sample. 
The second exploratory analysis was conducted to investigate is if there was a relationship between synchrony and CS type to SCR during recall. This analysis had a significant main effect of synchrony which showed increased synchrony being related to decreased SCR during recall, but not a significant synchrony by CS type interaction (Figure 2). It may be the case that youth overall learned that this was safe environment from their parent leading to overall decreases in arousal. Synchrony could also possibly be due to relationship quality between dyads. Relationship quality has previously been shown to be related to synchrony and therefore it is possible this is also related to overall lower arousal (Woody et al., 2016). In this pilot study, we were unable to collect parent-child relational measures, but future studies should consider adding these measures to parse apart why and how synchrony is related to overall arousal.

For our last exploratory analysis, we found a significant interaction between CS type and synchrony to predict expectancy of a stimulation, or the unconditioned stimulus (Figure 3). While the three CS types were significantly different from each other, we wanted to know if any of the CS types were different from zero. After running three linear models, only the CS- was marginally significant from zero. This, combined with our second exploratory analysis, may indicate the synchrony is more related to safety and overall arousal, than fear learning. If it was more related to fear learning, it would more likely be related to either just the CS+V or both the CS+D.

There are three limitations that must be considered in this pilot study. First, because of its nature as a pilot study, we have a small sample size. The original study was created to test feasibility of a three-day electrodermal stimulation fear extinction learning paradigm. We had minimal dropout and found that a three-day, instead of a two-day study, maintained extinction learning better in youth. Further, youth with PTSD is a difficult to recruit population. We hope these analyses will be used to guide future hypotheses, but all results in these preliminary analyses should be taken with caution.

Another limitation is the minimal parent psychopathology measures. It is well known in the literature that parent psychopathology and other parental factors including parenting style and relationship affect the child's affinity for psychopathology (Zhang et al., 2020). In this study we had the parents fill out the MINI, which gave a binary of current or lifetime DSM-IV psychopathology diagnoses. In the future, it would be beneficial to utilize psychopathology symptom severity scores as well as presence of specific diagnosis. Further, it would be useful to see if parent styles or parent-child relationships were related to synchrony.

Lastly, it is important to include trauma-exposed controls in addition to youth with PTSD and TD dyads. This will account for general effect of trauma and its relationship to synchrony and vicarious extinction learning. This also may answer potential questions about if parenting styles or relationships may buffer the effect of trauma and if this can be shown through synchrony. Futures studies should include this important group to disentangle trauma, formal diagnosis, and synchrony. 
317 This study gave us some of the first insight into potential mechanisms underlying 318 vicarious extinction learning in youth with PTSD. Learning fear and safety cues from 319 their caregivers may be disrupted in youth with a diagnosis of PTSD compared to their 320 TD counterparts, and this could further disrupt fear or safety learning in general. 
Asher, M., Barthel, A. L., Hofmann, S. G., Okon-Singer, H., \& Aderka, I. M. (2021). When two hearts beat as one: Heart-rate synchrony in social anxiety disorder. Behaviour Research and Therapy, 141, 103859. https://doi.org/10.1016/j.brat.2021.103859

Benedek, M., \& Kaernbach, C. (2010). Decomposition of skin conductance data by means of nonnegative deconvolution. Psychophysiology, 47(4), 647-658. https://doi.org/10.1111/j.1469-8986.2009.00972.x

Bilodeau-Houle, A., Bouchard, V., Morand-Beaulieu, S., Herringa, R. J., Milad, M. R., \& Marin, M.-F. (2020). Anxiety Sensitivity Moderates the Association Between Father-Child Relationship Security and Fear Transmission. Frontiers in Psychology, 11. https://doi.org/10.3389/fpsyg.2020.579514

Blechert, J., Michael, T., Vriends, N., Margraf, J., \& Wilhelm, F. H. (2007). Fear conditioning in posttraumatic stress disorder: Evidence for delayed extinction of autonomic, experiential, and behavioural responses. Behaviour Research and Therapy, 45(9), 2019-2033. https://doi.org/10.1016/j.brat.2007.02.012

Cheng, C. H., Lee, S. S., Lee, S.-K., Bray, C., Zimmerman, T., \& Gewirtz, A. H. (2021). A Conversion Crosswalk for the UCLA PTSD Reaction Index: Translating DSM-IV to DSM5. Journal of Traumatic Stress, 34(4), 872-879. https://doi.org/10.1002/jts.22694

Coco, M. I., \& Dale, R. (2014). Cross-recurrence quantification analysis of categorical and continuous time series: An R package. Frontiers in Psychology, 5. https://doi.org/10.3389/fpsyg.2014.00510

Danyluck, C., \& Page-Gould, E. (2019). Social and Physiological Context can Affect the Meaning of Physiological Synchrony. Scientific Reports, 9(1), 8222. https://doi.org/10.1038/s41598-019-44667-5

Davis, M., Bilms, J., \& Suveg, C. (2017). In Sync and in Control: A Meta-Analysis of ParentChild Positive Behavioral Synchrony and Youth Self-Regulation. Family Process, 56(4), 962-980. https://doi.org/10.1111/famp.12259

Debiec, J., \& Olsson, A. (2017). Social Fear Learning: From Animal Models to Human Function. Trends in Cognitive Sciences, 21(7), 546-555. https://doi.org/10.1016/j.tics.2017.04.010

Dunne, G., \& Askew, C. (2018). Vicarious learning and reduction of fear in children via adult and child models. Emotion, 18(4), 528-535. https://doi.org/10.1037/emo0000341

Faghih, R. T., Stokes, P. A., Marin, M.-F., Zsido, R. G., Zorowitz, S., Rosenbaum, B. L., Huijin Song, Milad, M. R., Dougherty, D. D., Eskandar, E. N., Widge, A. S., Brown, E. N., \& Barbieri, R. (2015). Characterization of fear conditioning and fear extinction by analysis of electrodermal activity. 2015 37th Annual International Conference of the IEEE Engineering in Medicine and Biology Society (EMBC), 7814-7818. https://doi.org/10.1109/EMBC.2015.7320204

Feldman, R. (2007). Parent-infant synchrony and the construction of shared timing; physiological precursors, developmental outcomes, and risk conditions. Journal of Child Psychology and Psychiatry, and Allied Disciplines, 48(3-4), 329-354. https://doi.org/10.1111/j.1469-7610.2006.01701.x

Feldman, R. (2012). Bio-behavioral Synchrony: A Model for Integrating Biological and Microsocial Behavioral Processes in the Study of Parenting. Parenting, 12(2-3), 154164. https://doi.org/10.1080/15295192.2012.683342

Garfinkel, S. N., Abelson, J. L., King, A. P., Sripada, R. K., Wang, X., Gaines, L. M., \& Liberzon, I. (2014). Impaired Contextual Modulation of Memories in PTSD: An fMRI and Psychophysiological Study of Extinction Retention and Fear Renewal. The Journal of Neuroscience: The Official Journal of the Society for Neuroscience, 34(40), 1343513443. https://doi.org/10.1523/JNEUROSCI.4287-13.2014

Goldstein, S., Field, T., \& Healy, B. T. (1989). Concordance of play behavior and physiology in preschool friends. Journal of Applied Developmental Psychology, 10(3), 337-351. https://doi.org/10.1016/0193-3973(89)90034-8 
Golkar, A., Haaker, J., Selbing, I., \& Olsson, A. (2016). Neural signals of vicarious extinction learning. Social Cognitive and Affective Neuroscience, 11(10), 1541-1549. https://doi.org/10.1093/scan/nsw068

Helpman, L., Marin, M.-F., Papini, S., Zhu, X., Sullivan, G. M., Schneier, F., Neria, M., Shvil, E., Malaga Aragon, M. J., Markowitz, J. C., Lindquist, M. A., Wager, T. D., Milad, M. R., \& Neria, Y. (2016). Neural changes in extinction recall following prolonged exposure treatment for PTSD: A longitudinal fMRI study. Neurolmage: Clinical, 12, 715-723. https://doi.org/10.1016/j.nicl.2016.10.007

Heyn, S.A., George, G.C., Hamm, E., Olsen, C., Harari, J.H., Marin, M., Milad, M.R., \& Herringa, R.J. (2022) (Submitted to American Journal of Psychiatry). Vicarious Extinction Learning Paradigm Validation in Parent-Child Dyads: A Pilot Study of Pediatric PTSD.

Herringa, R. J., Birn, R. M., Ruttle, P. L., Burghy, C. A., Stodola, D. E., Davidson, R. J., \& Essex, M. J. (2013). Childhood maltreatment is associated with altered fear circuitry and increased internalizing symptoms by late adolescence. Proceedings of the National Academy of Sciences of the United States of America, 110(47), 19119-19124. https://doi.org/10.1073/pnas.1310766110

Marin, M.-F., Bilodeau-Houle, A., Morand-Beaulieu, S., Brouillard, A., Herringa, R. J., \& Milad, M. R. (2020). Vicarious conditioned fear acquisition and extinction in child-parent dyads. Scientific Reports, 10(1), 17130. https://doi.org/10.1038/s41598-020-74170-1

Marusak, H. A., Hehr, A., Bhogal, A., Peters, C., ladipaolo, A., \& Rabinak, C. A. (2020). Alterations in Fear Extinction Neural Circuitry and Fear-Related Behavior Linked to Trauma Exposure in Children. Behavioural Brain Research, 112958. https://doi.org/10.1016/j.bbr.2020.112958

McLaughlin, K. A., Sheridan, M. A., Gold, A. L., Duys, A., Lambert, H. K., Peverill, M., Heleniak, C., Shechner, T., Wojcieszak, Z., \& Pine, D. S. (2016). Maltreatment Exposure, Brain Structure, and Fear Conditioning in Children and Adolescents. Neuropsychopharmacology, 41(8), 1956-1964. https://doi.org/10.1038/npp.2015.365

Milad, M. R., Orr, S. P., Lasko, N. B., Chang, Y., Rauch, S. L., \& Pitman, R. K. (2008). Presence and acquired origin of reduced recall for fear extinction in PTSD: Results of a twin study. Journal of Psychiatric Research, 42(7), 515-520. https://doi.org/10.1016/j.jpsychires.2008.01.017

Milad, M. R., Pitman, R. K., Ellis, C. B., Gold, A. L., Shin, L. M., Lasko, N. B., Zeidan, M. A., Handwerger, K., Orr, S. P., \& Rauch, S. L. (2009). Neurobiological basis of failure to recall extinction memory in posttraumatic stress disorder. Biological Psychiatry, 66(12), 1075-1082.

Milad, M. R., Rosenbaum, B. L., \& Simon, N. M. (2014). Neuroscience of fear extinction: Implications for assessment and treatment of fear-based and anxiety related disorders. Behaviour Research and Therapy, 62, 17-23. https://doi.org/10.1016/j.brat.2014.08.006

Milad, M. R., Wright, C. I., Orr, S. P., Pitman, R. K., Quirk, G. J., \& Rauch, S. L. (2007). Recall of fear extinction in humans activates the ventromedial prefrontal cortex and hippocampus in concert. Biological Psychiatry, 62(5), 446-454. https://doi.org/10.1016/j.biopsych.2006.10.011

Motsan, S., Bar-Kalifa, E., Yirmiya, K., \& Feldman, R. (2020). Physiological and social synchrony as markers of PTSD and resilience following chronic early trauma. Depression and Anxiety. https://doi.org/10.1002/da.23106

Pärnamets, P., Espinosa, L., \& Olsson, A. (2020). Physiological synchrony predicts observational threat learning in humans. Proceedings of the Royal Society B: Biological Sciences, 287(1927), 20192779. https://doi.org/10.1098/rspb.2019.2779 
Pitman, R. K., Rasmusson, A. M., Koenen, K. C., Shin, L. M., Orr, S. P., Gilbertson, M. W., Milad, M. R., \& Liberzon, I. (2012). Biological studies of post-traumatic stress disorder. Nature Reviews. Neuroscience, 13(11), 769-787. https://doi.org/10.1038/nrn3339

Rachman, S. (1977). The conditioning theory of fearacquisition: A critical examination. Behaviour Research and Therapy, 15(5), 375-387. https://doi.org/10.1016/00057967(77)90041-9

RSTudio Team. (2012). RStudio: Integrated Development for R. RStudio, Inc.

Schiller, D., Levy, I., Niv, Y., LeDoux, J. E., \& Phelps, E. A. (2008). From fear to safety and back: Reversal of fear in the human brain. The Journal of Neuroscience: The Official Journal of the Society for Neuroscience, 28(45), 11517-11525. https://doi.org/10.1523/JNEUROSCI.2265-08.2008

Sheehan, D. V., Lecrubier, Y., Sheehan, K. H., Amorim, P., Janavs, J., Weiller, E., Hergueta, T., Baker, R., \& Dunbar, G. C. (1998). The Mini-International Neuropsychiatric Interview (M.I.N.I.): The development and validation of a structured diagnostic psychiatric interview for DSM-IV and ICD-10. The Journal of Clinical Psychiatry, 59 Suppl 20, 2233;quiz 34-57.

Woody, M. L., Feurer, C., Sosoo, E. E., Hastings, P. D., \& Gibb, B. E. (2016). Synchrony of physiological activity during mother-child interaction: Moderation by maternal history of major depressive disorder. Journal of Child Psychology and Psychiatry, 57(7), 843-850. https://doi.org/10.1111/jcpp.12562

Zhang, H., Lee, Z. X., White, T., \& Qiu, A. (2020). Parental and social factors in relation to child psychopathology, behavior, and cognitive function. Translational Psychiatry, 10(1), 1-9. https://doi.org/10.1038/s41398-020-0761-6 


\begin{tabular}{|c|c|c|c|c|}
\hline & & \begin{tabular}{|l} 
Typically \\
Developin \\
g
\end{tabular} & PTSD & $\begin{array}{l}\text { Group } \\
\text { Comparisons }\end{array}$ \\
\hline \multicolumn{5}{|c|}{ Basic Demographic Variables } \\
\hline N Dyads & & 5 & 11 & \\
\hline $\begin{array}{l}\text { Child Sex } \\
\text { (Female) }\end{array}$ & & 4 & 10 & $\begin{array}{l}c^{2}(1, N=16)= \\
0.37, p=0.54\end{array}$ \\
\hline $\begin{array}{l}\text { Parent Sex } \\
\text { (Female) }\end{array}$ & & 5 & 11 & $\begin{array}{l}c^{2}(1, \mathrm{~N}=16)= \\
0.48, p=0.49\end{array}$ \\
\hline Child Age & & 11.19 & 13.86 & $\begin{array}{l}t(15)=-1.31, p= \\
.21\end{array}$ \\
\hline Parent Age & & 44.74 & 41.71 & $t(14)=.75, p=.47$ \\
\hline $\begin{array}{l}\text { Did Parent } \\
\text { Receive a } \\
\text { Shock (No) }\end{array}$ & & 7 & 1 & $\begin{array}{l}c^{2}(1, N=15)= \\
1.64, p=0.20\end{array}$ \\
\hline \multirow[t]{7}{*}{ Race } & White & 4 & 7 & \\
\hline & African American & 0 & 1 & \\
\hline & Asian & 0 & 0 & \\
\hline & Two or More & 1 & 1 & \\
\hline & $\begin{array}{l}\text { Hispanic or } \\
\text { Latino }\end{array}$ & 0 & 2 & \\
\hline & $\begin{array}{l}\text { Not Hispanic } \\
\text { Latino }\end{array}$ & 5 & 7 & \\
\hline & Not Provided & 0 & 2 & \\
\hline \multirow{6}{*}{$\begin{array}{l}\text { Parent } \\
\text { Education } \\
\text { Level }\end{array}$} & $\begin{array}{l}\text { Some High } \\
\text { School }\end{array}$ & 0 & 0 & \\
\hline & $\begin{array}{l}\text { High School } \\
\text { Degree }\end{array}$ & 0 & 3 & \\
\hline & Some College & 1 & 2 & \\
\hline & College Degree & 1 & 3 & \\
\hline & Graduate Degree & 3 & 0 & \\
\hline & Not Provided & 0 & 3 & \\
\hline \multicolumn{5}{|c|}{ Trauma Variables } \\
\hline PTSD-RI Total & & $8.75(8.32)$ & \begin{tabular}{|l|}
31.09 \\
$(9.47)$
\end{tabular} & $\begin{array}{l}t(13)=-3.88 \\
p=.002\end{array}$ \\
\hline $\begin{array}{l}\text { PTSD-RI } \\
\text { Reexperiencin } \\
\text { g }\end{array}$ & & $2(2.92)$ & $9.09(4.29)$ & $\begin{array}{l}t(13)=-2.85 \\
p=.014\end{array}$ \\
\hline
\end{tabular}




\begin{tabular}{|c|c|c|c|}
\hline $\begin{array}{l}\text { PTSD-RI } \\
\text { Avoidance }\end{array}$ & $2.5(1.66)$ & $10.82(4.93$ & $\begin{array}{l}t(13)=-3.08 \\
p=.009\end{array}$ \\
\hline $\begin{array}{l}\text { PTSD-RI } \\
\text { Hyperarousal }\end{array}$ & $4.25(3.96)$ & $\begin{array}{l}11.18(4.32 \\
)\end{array}$ & $\begin{array}{l}t(13)=-2.61 \\
p=.022\end{array}$ \\
\hline MFQ & - & $19.1(8.97)$ & \\
\hline SCARED & - & $32.5(11.91$ & \\
\hline $\begin{array}{l}\text { Parent Current } \\
\text { Diagnosis } \\
\text { (Presence) }\end{array}$ & 1 & 5 & $\begin{array}{l}c^{2}(1, \mathrm{~N}=13)= \\
2.24, p=0.13\end{array}$ \\
\hline $\begin{array}{l}\text { Parent Lifetime } \\
\text { Diagnosis } \\
\text { (Presence) }\end{array}$ & 2 & 5 & $\begin{array}{l}c^{2}(1, \mathrm{~N}=13)= \\
0.63, p=0.43\end{array}$ \\
\hline \multicolumn{4}{|c|}{$\begin{array}{l}\text { Table 1: Full Sample Participant Demographics. Both parent and youth groups did } \\
\text { not differ significantly in sex or age. There was a significant difference between } \\
\text { groups for the PTSD-RI but did not differ on parent diagnoses. }\end{array}$} \\
\hline \multicolumn{4}{|c|}{$\begin{array}{l}\text { Abbreviations: PTSD, Posttraumatic Stress Disorder; PTSD-RI, PTSD-Reaction } \\
\text { Index; MFQ, Mood and Feelings Questionnaire; SCARED, Screen for Child Anxiety- } \\
\text { Related Mood Disorders. }\end{array}$} \\
\hline
\end{tabular}

